\title{
Influência das condições de processamento cerâmico na resistência mecânica e na permeabilidade dos filtros de $\mathrm{Al}_{2} \mathrm{O}_{3}-\mathrm{SiC}$
}

\section{(Influence of ceramic processing on the mechanical resistance and permeability of filters in the $\mathrm{Al}_{2} \mathrm{O}_{3}$-SiC system)}

\author{
V. R. Salvini, M. D. M. Innocentini, V. C. Pandolfelli \\ Departamento de Engenharia de Materiais - DEMa \\ Universidade Federal de S. Carlos - UFSCar \\ Rod. Washington Luiz, km 235, C.P. 676, 13565-905, S. Carlos, SP \\ pvrs@iris.ufscar.br,vicpando@power.ufscar.br
}

\begin{abstract}
Resumo
Filtros cerâmicos devem apresentar alta permeabilidade e eficiência de retenção de inclusões sólidas, além de uma boa resistência mecânica. No entanto, estas propriedades variam de modos distintos para uma determinada estrutura celular. Poros grandes aumentam a permeabilidade, mas diminuem a eficiência de retenção das inclusões. Em relação ao desempenho do filtro, a porosidade aparente apresenta alta relevância, uma vez que a resistência mecânica diminui e a permeabilidade aumenta para valores de porosidade elevados. Neste trabalho investigou-se a relação entre a resistência mecânica e a permeabilidade para filtros do sistema $\mathrm{Al}_{2} \mathrm{O}_{3}-\mathrm{SiC}$ de $10 \mathrm{ppi}$ (poros por polegada linear). A quantidade da suspensão impregnada na esponja, durante a fabricação do filtro, foi escolhida como variável de controle, pois por meio desta modifica-se a porosidade e o tamanho de poro do filtro. Os resultados obtidos foram comparados aos de filtros de 8 a 90 ppi.

Palavras-chave: filtros cerâmicos, processamento cerâmico, resistência mecânica, permeabilidade.
\end{abstract}

\begin{abstract}
Ceramic filters must present not only high permeability and particle trapping efficiency, but also suitable mechanical strength. However, these parameters are influenced in different ways by the cellular structure. Large pores favor permeability, but lower removal efficiency of small particles. Small pores, on the other hand, enhance particle collection, although the filter pressure drop increases. The porosity is also essential for determining ceramic foam performance. Mechanical strength generally decreases with increasing porosity, even though permeability is improved. In this work, the relationship between mechanical strength and permeability has been investigated for 10 ppi (pores per linear inch) $\mathrm{Al}_{2} \mathrm{O}_{3}$-SiC filters. The amount of slurry impregnated on the organic struts walls during processing has been chosen as the control variable. The results have been compared with those obtained from cellular structures where the pore count variation ranged from 10 to 90 ppi.
\end{abstract}

Keywords: ceramic filters, ceramic processing, mechanical strength, permeability.

\section{INTRODUÇÃO}

A técnica mais comum de produção de filtros cerâmicos é o método da réplica, que envolve o recobrimento de uma esponja polimérica de células abertas com uma suspensão cerâmica. A composição química da suspensão depende da aplicação do filtro. Após as etapas de calcinação para eliminação do polímero e da sinterização, tem-se uma réplica da esponja em cerâmica [1].

Normalmente, as propriedades estruturais do filtro cerâmico produzido por esta técnica são controladas pelas características da esponja polimérica. Estas propriedades incluem a porosidade, o tamanho de poro e o número de poros por polegada linear (ppi) [2].
Em uso os filtros devem apresentar não somente alta permeabilidade e eficiência de retenção de inclusões sólidas, mas também boa resistência mecânica. Estes parâmetros são influenciados de modos distintos para uma determinada estrutura celular. Poros grandes favorecem a permeabilidade, mas reduzem a eficiência de coleta das inclusões. Poros pequenos, por sua vez, aumentam a eficiência de retenção das inclusões sólidas, mas também aumentam a queda de pressão através do filtro [3].

Além do tamanho de poro, o controle da porosidade também é essencial na avaliação do desempenho do filtro. A resistência mecânica de cerâmicas porosas geralmente diminui com o 
aumento da porosidade, ao passo que a permeabilidade aumenta $[4,5]$. A melhoria na resistência mecânica de filtros cerâmicos produzidos pelo método da réplica pode ser obtida pela densificação dos filamentos ou pelo entupimento dos poros da estrutura celular.

Assim, o desenvolvimento de um método capaz de modificar a estrutura celular dos filtros, por meio da variação da porosidade e do tamanho de poro, é desejável para se adequar suas propriedades à aplicação.

Há dois métodos tradicionais de modificação celular de filtros: o primeiro dá-se através da variação do número de poros por polegada linear (ppi) da esponja polimérica precursora e, o segundo, pela variação da quantidade de suspensão impregnada na esponja durante a fabricação dos filtros.

Segundo a literatura $[2,6]$, pelo primeiro método modificase o tamanho de poro do filtro, mas a porosidade permanece praticamente constante. Tal comportamento é decorrente do fato de que todas as esponjas poliméricas apresentam porosidade em torno de $95 \%$, independentemente do ppi. Assim, o processamento cerâmico apenas recobre os filamentos da esponja, alterando proporcionalmente sua porosidade.

Neste trabalho investigou-se o segundo método de modificação da estrutura celular de filtros cerâmicos do sistema $\mathrm{Al}_{2} \mathrm{O}_{3}$-SiC. Para isto, a quantidade da suspensão cerâmica impregnada em uma esponja polimérica de $10 \mathrm{ppi}$, durante a fabricação dos filtros, foi escolhida como variável de controle. Os resultados de resistência mecânica e permeabilidade dos filtros produzidos foram correlacionados e um método de avaliar o desempenho destes materiais foi proposto por meio de um novo parâmetro, denominado parâmetro de otimização. Os resultados obtidos foram comparados aos dos filtros de $\mathrm{Al}_{2} \mathrm{O}_{3}-\mathrm{SiC}$ produzidos com variação no ppi da esponja na faixa de 8 a 90 ppi.

\section{MATERIAIS E MÉTODOS}

Para este estudo foram produzidas amostras cilíndricas de filtros (diâmetro de $7 \mathrm{~cm}$ e espessura igual a $2,5 \mathrm{~cm}$ ), onde a mudança das propriedades estruturais (porosidade e tamanho de poro) foi obtida via modificações no processamento cerâmico.

Utilizando-se uma suspensão aquosa de $\mathrm{Al}_{2} \mathrm{O}_{3}-\mathrm{SiC}$ com $58 \%$-v de sólidos, produziu-se filtros de 10 ppi por meio da variação da abertura da calandra na faixa de 10 a $40 \%$ da espessura da esponja, para uma e duas impregnações.

A calandra é constituída de dois roletes dispostos paralelamente entre si, sendo a distância entre ambos conhecida como abertura da calandra. O ajuste desta abertura é efetuado tomando-se como referência à espessura da esponja polimérica, uma vez que a função da etapa de calandragem é retirar o excesso da suspensão cerâmica da esponja por compressão da mesma entre os roletes. Assim, quanto maior for a abertura da calandra uma quantidade maior da suspensão cerâmica ficará retida na esponja polimérica aumentando, desse modo, a espessura dos filamentos.

Após a impregnação e calandragem das esponjas poliméricas, estas foram secas em três etapas: ao ar por 24 horas, em câmara climática (Vötsch, modelo 20-20) a $40{ }^{\circ} \mathrm{C}$ com $70 \%$ de umidade relativa e, finalmente, em estufa a $100{ }^{\circ} \mathrm{C}$ por 24 horas. Após a secagem, as amostras foram calcinadas a $400{ }^{\circ} \mathrm{C} / 1 \mathrm{~h}$ e a $900{ }^{\circ} \mathrm{C} / 1 \mathrm{~h}$, com taxa de aquecimento de $2{ }^{\circ} \mathrm{C} /$ min para eliminação das esponjas poliméricas. Finalmente, os filtros foram sinterizados a $1250{ }^{\circ} \mathrm{C}$ por 5 horas ao ar.

Após a produção dos filtros, estes foram caracterizados quanto às suas propriedades físicas (tamanho de poro, densidade, porosidade e número de poros por polegada linear), fluidodinâmicas (constantes de permeabilidade Darciana, $\mathrm{k}_{1}$, e não-Darciana, $\mathrm{k}_{2}$ ) e mecânica (resistência mecânica sob compressão uniaxial).

\section{Caracterização física}

A fração de vazios no filtro determina sua porosidade aparente $\left(\varepsilon_{\mathrm{ap}}\right)$, expressa por $\left[1-\left(\rho / \rho_{\mathrm{s}}\right)\right]$. A densidade geométrica $(\rho)$ foi medida a partir das dimensões e massa dos corpos-deprova e, a densidade real $\left(\rho_{\mathrm{s}}\right)$, foi determinada por picnometria de hélio dos filamentos moídos (Accupyc, Micromeritics). $\mathrm{O}$ diâmetro de poro (d) foi determinado por análise de imagens utilizando-se o software Image-Pro Plus 3.0.

\section{Caracterização fluidodinâmica.}

Após a caracterização física, os filtros foram analisados quanto à permeabilidade em um equipamento hidrodinâmico desenvolvido em laboratório [6]. Neste equipamento, os dados de queda de pressão $(\Delta \mathrm{P})$ através do filtro de espessura $\mathrm{L}$ foram coletados em função da velocidade superficial de escoamento $\left(\mathrm{v}_{\mathrm{s}}\right.$ ) do fluido utilizado (água). Para cada amostra foram feitas três repetições (ensaio, réplica e tréplica) para confirmação dos resultados. Após a coleta de dados, calculou-se as constantes de permeabilidade $k_{1}$ e $k_{2}$ por meio do ajuste da equação de Forchheimer [6], expressa por:

$$
\frac{\Delta \mathrm{P}}{\mathrm{L}}=\frac{\mu}{\mathrm{k}_{1}} \mathrm{v}_{\mathrm{s}}+\frac{\rho}{\mathrm{k}_{2}} \mathrm{v}_{\mathrm{s}}^{2}
$$

aos dados experimentais de queda de pressão $(\Delta \mathrm{P} / \mathrm{L})$ em função da velocidade de escoamento $\left(\mathrm{v}_{\mathrm{s}}\right)$. Na equação $(\mathrm{A})$ os parâmetros $\mu$ e $\rho$ correspondem, respectivamente, a viscosidade e densidade da água, calculadas em função da temperatura do fluido e da pressão atmosférica de cada ensaio. Os termos da equação $(\mathrm{A})$ correspondem às contribuições viscosas (Darciana) e inerciais (não-Darciana) à queda de pressão total do fluido. A predominância de um ou outro termo na queda de pressão para um dado fluido é função da velocidade utilizada e das constantes $\mathrm{k}_{1}$ e $\mathrm{k}_{2}$. A temperatura da água, a vazão volumétrica e a pressão durante os ensaios variaram de 29 e $35^{\circ} \mathrm{C}$, de 0 a $14 \mathrm{~m}^{3} / \mathrm{s}$ e 0 a $45 \times 10^{3} \mathrm{~Pa} / \mathrm{m}$, respectivamente.

\section{Caracterização mecânica.}

Após o ensaio de permeabilidade as amostras foram secas em estufa a $100^{\circ} \mathrm{C} / 24 \mathrm{~h}$ e, em seguida, ensaiadas quanto à resistência mecânica sob compressão utilizando-se uma máquina de ensaios universal MTS 810 com velocidade do 
atuador de 1,3 mm/min. As superfícies da amostra ortogonais à aplicação da carga de compressão foram cobertas com uma borracha semi-rígida. Isso é recomendado para eliminar os efeitos de carregamento localizado, devido à topografia da superfície das amostras [6].

\section{RESULTADOS E DISCUSSÃO}

A variação na abertura da calandra no processamento provocou grandes modificações nas propriedades físicas dos filtros de $\mathrm{Al}_{2} \mathrm{O}_{3}-\mathrm{SiC}$ de 10 ppi, conforme ilustrado na Fig. 1.

Na Fig. 1 (a) a diminuição da porosidade aparente $\left(\varepsilon_{\text {ap }}\right)$ é decorrente do aumento da massa da suspensão cerâmica retida na esponja quando se aumenta a abertura da calandra. O decréscimo do número total de poros é consequiência do entupimento dos poros de menor tamanho obtido com a abertura da calandra (Fig. 1 (b)). Conseqüentemente, tem-se um deslocamento da curva de distribuição de diâmetro de poro para valores maiores, explicando o aumento de $\overline{d_{p}}$ na Fig. 1 (c). Observa-se também na Fig. 1 que não houve diferença significativa nos resultados obtidos para uma e duas impregnações.

Observa-se na Fig. 2 que a resistência mecânica, $\sigma_{c}$, aumentou exponencialmente com o aumento da abertura da
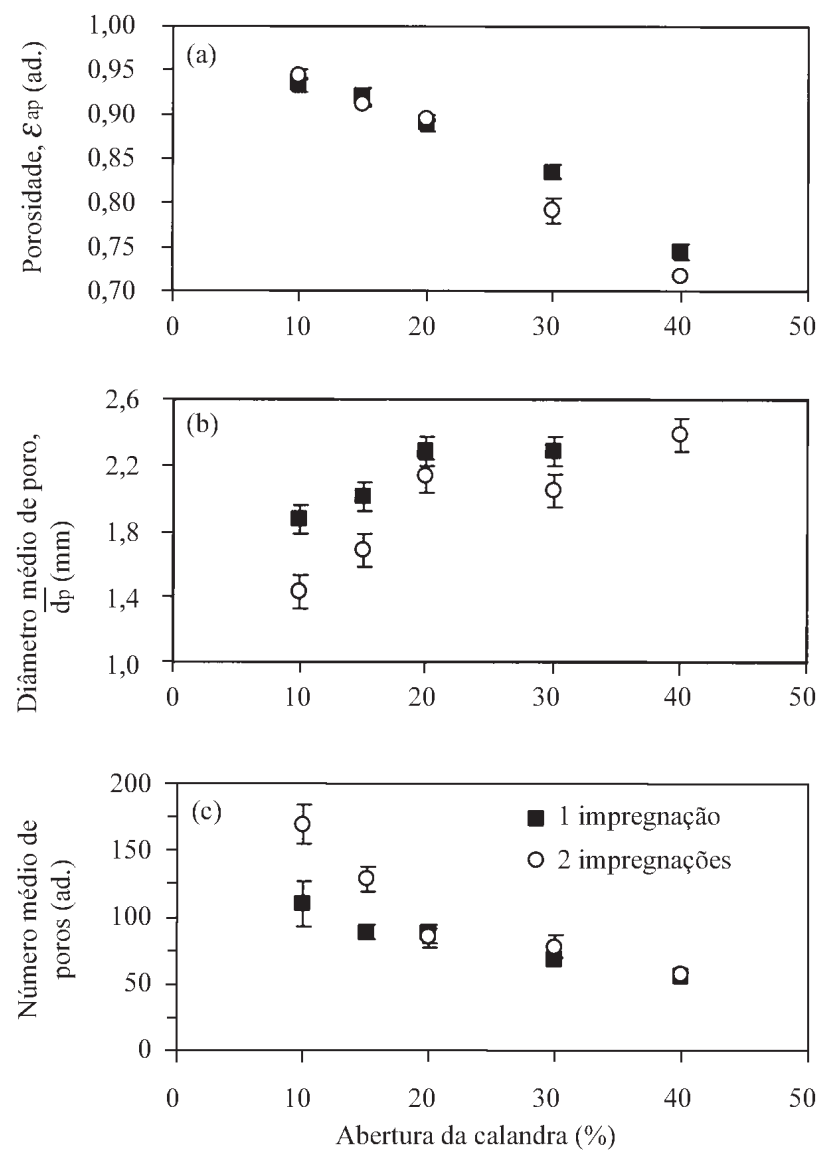

Figura 1: Influência da abertura da calandra nas propriedades estruturais dos filtros do sistema $\mathrm{Al}_{2} \mathrm{O}_{3}-\mathrm{SiC}$ de 10 ppi: (a) porosidade, $\varepsilon_{\mathrm{ap}}$, (b) diâmetro médio de poro, $\overline{\mathrm{d}}_{\mathrm{p}} \mathrm{e}$ (c) número médio de poros.

[Figure 1: Influence of foam compression on the structural properties of 10 ppi filters in the $\mathrm{Al}_{2} \mathrm{O}_{3}$-SiC system: (a) porosity, $\varepsilon_{a p}$, (b) average pore size, $\bar{d}_{p}$ and (c) average pore number.] calandra em decorrência da diminuição da porosidade e do maior espessamento dos filamentos dos poros.

Os resultados das constantes de permeabilidade para os filtros de 10 ppi foram também bastante sensíveis às mudanças da estrutura celular dos filtros. As constantes de permeabilidade Darciana, $\mathrm{k}_{1}$, e não-Darciana, $\mathrm{k}_{2}$, tiveram uma redução de aproximadamente duas ordens de magnitude quando a abertura da calandra aumentou de 10 para $40 \%$ da espessura da esponja. A diminuição nos níveis de permeabilidade pode ser também atribuída à redução da porosidade e à diminuição do caminho livre para o escoamento do fluido, causado pelo espessamento dos filamentos na estrutura celular.

Contudo, cabe mencionar que propriedades estruturais dos filtros $\left(\varepsilon_{\text {ap }}\right.$ e $\left.d_{p}\right)$ influenciam as constantes de permeabilidade, $\mathrm{k}_{1}$
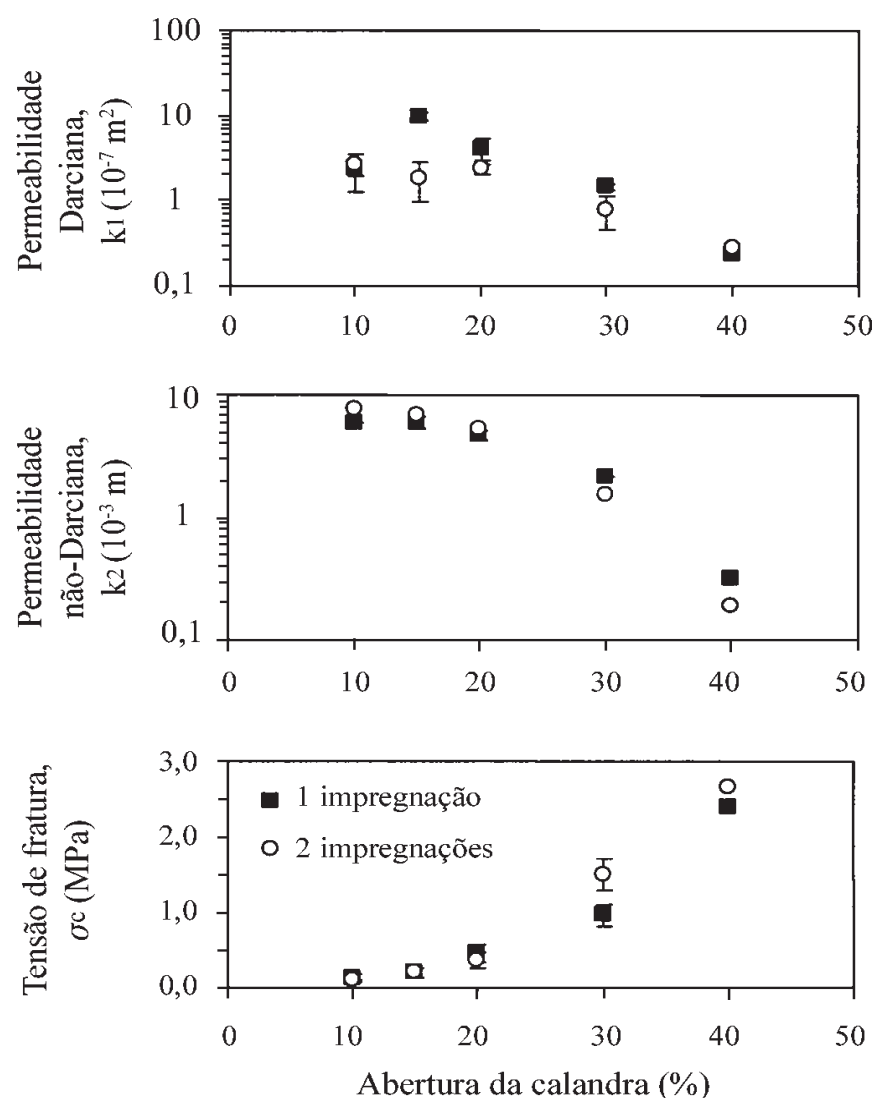

Figura 2: Influência da abertura da calandra nas propriedades fluidodinâmicas e mecânicas dos filtros de $\mathrm{Al}_{2} \mathrm{O}_{3}$-SiC de 10 ppi. [Figure 2: Influence of foam compression on the flow-dynamic and mechanical properties of 10 ppi filters in the $\mathrm{Al}_{2} \mathrm{O}_{3}$-SiC system.]

e $\mathrm{k}_{2}$, de maneira distinta. A redução da permeabilidade Darciana, $\mathrm{k}_{1}$, observada na Fig. 2, é decorrente da intensificação do contato fluido-sólido que acontece quando a porosidade aparente $\left(\varepsilon_{\text {ap }}\right)$ do filtro diminui. Isto resulta em uma maior área disponível para o atrito e, conseqüentemente, maior perda de pressão pelo fluido.

Já a permeabilidade não-Darciana, $\mathrm{k}_{2}$, representa as perdas inerciais durante o escoamento do fluido através do meio poroso, indicado pelo produto $\left(\rho \cdot \mathrm{v}_{\mathrm{s}}^{2}\right)$. Neste caso, as alterações estão associadas à turbulência do escoamento e tortuosidade do meio [2]. Desse modo, o comportamento da constante de 
permeabilidade não-Darciana, $\mathrm{k}_{2}$, é de queda em relação à diminuição da porosidade aparente $\left(\varepsilon_{\text {ap }}\right)$ do filtro, uma vez que o trajeto a ser permeado pelo fluido torna-se mais estreito e tortuoso, causando o aumento da velocidade intersticial dentro do meio poroso e, conseqüentemente, a perda de energia inercial.

Mas, qual seria a abertura de calandra mais adequada para a produção de filtros cerâmicos de $\mathrm{Al}_{2} \mathrm{O}_{3}-\mathrm{SiC}$ de 10 ppi simultaneamente resistentes e permeáveis? Com o intuito de correlacionar a condição de processamento cerâmico do filtro ao seu desempenho fluidodinâmico e mecânico, foi proposto neste trabalho um parâmetro de otimização, P.O., expresso por:

$$
\text { P.O. }=\frac{\sigma_{\mathrm{i}} \cdot \Delta \mathrm{P}_{\operatorname{mim}}}{\sigma_{\max } \cdot \Delta \mathrm{P}_{\mathrm{i}}}
$$

onde $\sigma_{\mathrm{i}} \mathrm{e} \Delta \mathrm{P}_{\mathrm{i}}$ correspondem, respectivamente, à resistência mecânica sob compressão e à queda de pressão para a amostra $\mathrm{i}$ a uma dada velocidade de escoamento, $\mathrm{v}_{\mathrm{s}}$. Os parâmetros $\sigma_{\text {máx }}$ e $\Delta \mathrm{P}_{\text {min }}$, representam, por sua vez, a resistência mecânica máxima e a queda de pressão mínima dentro do conjunto de amostras analisadas.

O parâmetro P.O. pode variar de 0 a 1 . O valor de P.O. máximo indica que a melhor combinação entre a permeabilidade e a resistência mecânica foi obtida.

Com o intuito de avaliar o comportamento dos filtros
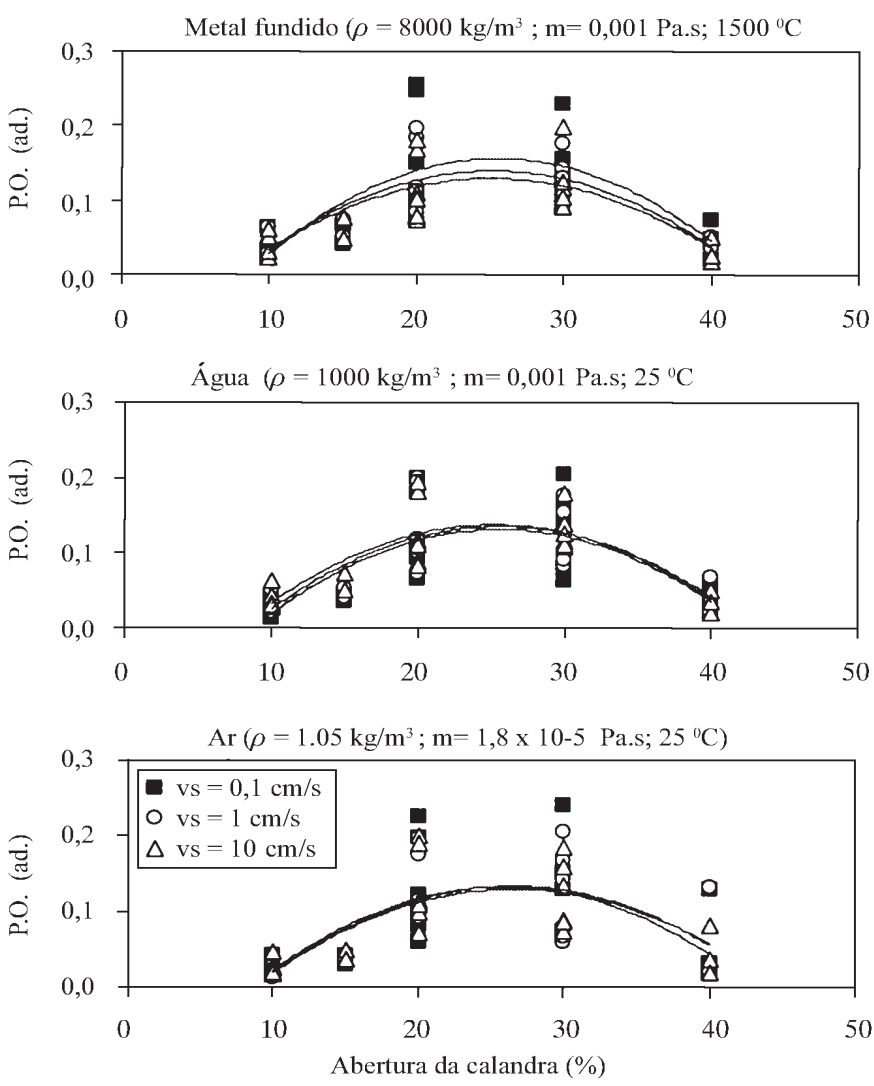

Figura 3: Parâmetro de otimização, P.O., calculado para os filtros de $\mathrm{Al}_{2} \mathrm{O}_{3}$-SiC de 10 ppi produzidos com diferentes aberturas de calandra. [Figure 3: Optimization parameter, O.P., evaluated for 10 ppi filters in the $\mathrm{Al}_{2} \mathrm{O}_{3}$-SiC system, produced with different level of foam compression.]
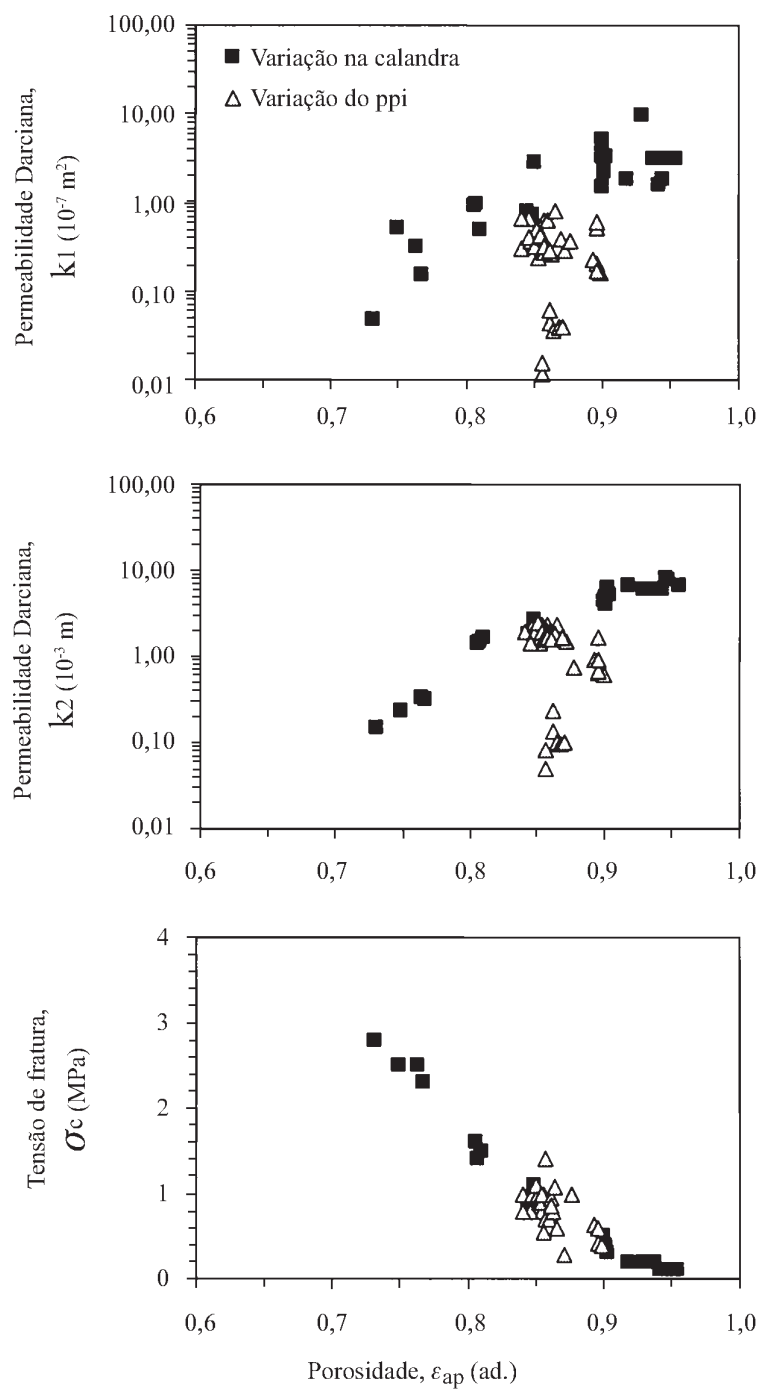

Figura 4: Influência da porosidade aparente, $\varepsilon_{\mathrm{ap}}$, nas constantes de permeabilidade, $\mathrm{k}_{1}$ e $\mathrm{k}_{2}$, e na resistência mecânica sob compressão, $\sigma$, dos filtros do sistema $\mathrm{Al}_{2} \mathrm{O}_{3}-\mathrm{SiC}$.

[Figure 4: Influence of aparent porosity, $\mathcal{E}_{a p}$, on the permeability constants, $k_{1}$ and $k_{2}$, and on the compressive mechanical resistance, $\sigma_{e}$, for filters in the $\mathrm{Al}_{2} \mathrm{O}_{3}$-SiC system.]

produzidos para o escoamento de diferentes fluidos, estimouse o parâmetro de otimização P.O. para os filtros de 10 ppi considerando-se como fluidos de teste o ar, a água e um metal fundido. Ainda no cálculo de P.O., considerou-se uma faixa ampla de velocidade de escoamento do fluido $(0,1$ a $10 \mathrm{~cm} / \mathrm{s})$ com o intuito de observar a influência dos efeitos viscosos e inerciais na curva de queda de pressão $\Delta \mathrm{P}$. As curvas obtidas são ilustradas na Fig. 3.

Apesar do espalhamento dos dados na Fig. 3, os resultados revelam que o tipo de fluido e a velocidade de escoamento do mesmo não influenciaram de modo significativo o parâmetro de otimização, P.O.. Na prática, este resultado indica que as tendências observadas para P.O. em ensaios de escoamento com água podem ser, razoavelmente, extrapoladas para condições de filtração de outros fluidos, por exemplo, metais fundidos e aerossóis.

Observa-se também na Fig. 3 que, para todos os casos analisados, a melhor combinação de resistência mecânica e 
permeabilidade foi obtida para os filtros de 10 ppi produzidos com abertura de calandra entre 20 e $30 \%$ da espessura da esponja.

Ainda em relação à Fig. 3, a baixa resistência mecânica dos filamentos dos filtros produzidos com abertura de calandra de $10 \%$, foi responsável pela redução de P.O.. Por outro lado, a baixa permeabilidade dos filtros fabricados com abertura de calandra de $40 \%$ também reduziu o parâmetro de otimização, P.O..

A análise geral dos resultados apresentados permite concluir que, mesmo considerando que a resistência mecânica dos filtros cerâmicos também depende da composição química do material, o controle da quantidade da suspensão impregnada na esponja é uma importante ferramenta na otimização das propriedades fluidodinâmicas e mecânicas durante o processamento destes filtros.

As diferenças entre os dois métodos de modificação da estrutura celular dos filtros do sistema $\mathrm{Al}_{2} \mathrm{O}_{3}-\mathrm{SiC}$ (ppi da esponja precursora e massa retida no processamento) tornam-se evidentes quando comparam-se as mudanças nas propriedades estruturais dos filtros $\left(\varepsilon_{\text {ap }}\right.$ e $\left.d_{p}\right)$, conforme observado nas Figs. 4 e 5 , respectivamente.
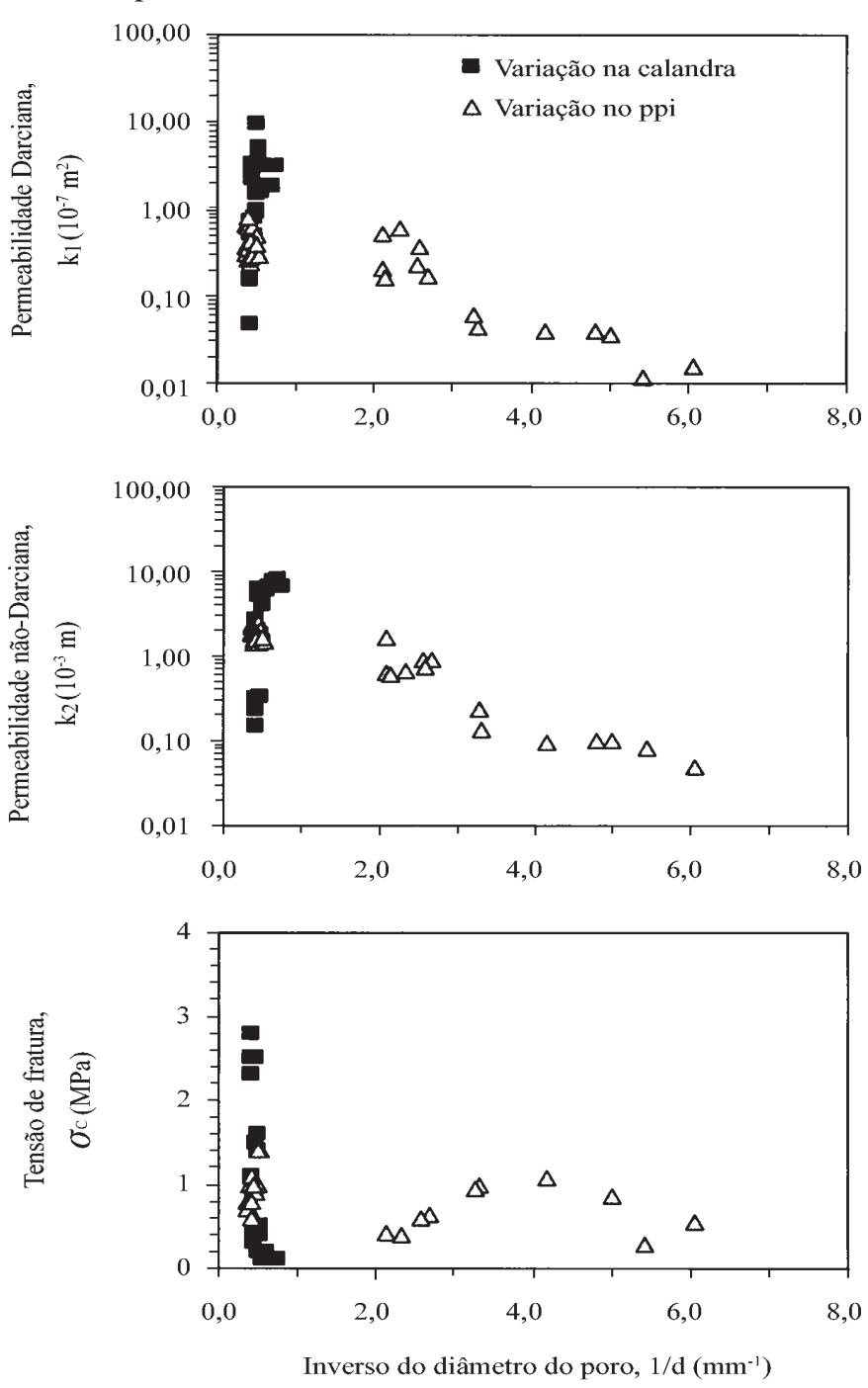

Figura 5: Influência do tamanho de poro, $\mathrm{d}_{\mathrm{p}}$, sobre as propriedades fluidodinâmicas, $\mathrm{k}_{1}$ e $\mathrm{k}_{2}$, e mecânicas, $\sigma_{c}$, dos filtros do sistema $\mathrm{Al}_{2} \mathrm{O}_{3}-\mathrm{SiC}$. [Figura 5: Influence of pore size, $d_{p}$, on the flow-dynamic properties, $k_{1}$ and $k_{2}$, and mechanical, $\sigma_{c^{\prime}}$ for filters in the $\mathrm{Al}_{2} \mathrm{O}_{3}$-SiC system.]
Na Fig. 4 observa-se que o método de alterar a estrutura celular por meio da variação na abertura da calandra tem uma relação bem definida com a porosidade aparente $\left(\varepsilon_{\text {ap }}\right)$ dos filtros e, esta por sua vez, alterou sensivelmente a permeabilidade e a resistência mecânica desses materiais. Já a variação no ppi da esponja precursora produziu filtros com porosidade em uma estreita faixa $(0,85$ a 0,90$)$, concordando com os resultados da literatura $[2,6]$.

Já na Fig. 5 nota-se que o tamanho de poro do filtro $\left(d_{p}\right)$ foi predominantemente influenciado pelo ppi da esponja precursora. Nota-se também que não houve uma influência clara do tamanho de poro do filtro na sua resistência mecânica. Tal comportamento deve estar associado à etapa do processamento cerâmico, uma vez que, diferentemente das propriedades fluidodinâmicas, a resistência mecânica destes materiais é fortemente afetada pela presença de defeitos.

\section{CONCLUSÕES}

Com relação às modificações estruturais nos filtros cerâmicos decorrente do processamento e das características da espuma polimérica, os resultados indicaram que a variação na abertura da calandra produziu estruturas celulares com uma larga faixa de porosidade e distribuição de tamanho de poro estreita, afetando de modo proporcional a resistência mecânica e permeabilidade dos filtros. De modo complementar, a variação no ppi da esponja resultou em modificações nessas propriedades físicas com melhor correlação com o tamanho de poro $\left(d_{p}\right)$. O parâmetro de otimização P.O. proposto neste trabalho permitiu identificar a melhor condição de processamento para se otimizar simultaneamente o desempenho fluidodinâmico e mecânico dos filtros de 10 ppi. De uma maneira geral, observou-se que a associação dos dois métodos de modificação estrutural - variação do ppi da esponja e variação da massa retida - constitui-se uma ferramenta importante no projeto de cerâmicas porosas para aplicações envolvendo a mistura e filtragem de fluidos.

\section{AGRADECIMENTOS}

Os autores deste trabalho agradecem à FAPESP pelo apoio dado a este trabalho e à Alcoa Alumínio S. A. pelo fornecimento das matérias-primas.

\section{REFERÊNCIAS}

[1] J. Saggiowoyansky, C. E. Scott, W. P. Minnear, Am. Ceram. Soc. Bull. 71, 11 (1992) 1674-1682.

[2] M. D. M. Innocentini, V. R. Salvini, V. C. Pandolfelli, J. R. Coury, Am. Ceram. Soc. Bull. 78, 9 (1999) 78-84.

[3] V. R. Salvini, M. D. M. Innocentini, V. C. Pandolfelli, Ceram. News 7, 1 (2000) 49-54.

[4] V. R. Salvini, M. D. M. Innocentini, V. C. Pandolfelli, Am. Ceram. Soc. Bull. 79, 5 (2000) 49-54.

[5] M. D. M. Innocentini, P. Sepúlveda, V. R. Salvini, V. C. Pandolfelli, J. Am. Ceram. Soc. 81, 12 (1998) 3349-3352.

[6] V. R. Salvini, Tese de Doutorado, UFSCar, DEMa, PPGCEM (2001).

(Rec. 04/03/02, Rev. 20/04/02, Ac. 20/04/02) 